\title{
NANOARRAY-ENHANCED IMPLANTABLE INTRAOCULAR PRESSURE SENSOR WITH REMOTE OPTICAL READOUT
}

\author{
Jeong Oen Lee ${ }^{*}$, Kun Huang ${ }^{1}$, Trong-Tuong Nguyen ${ }^{2}$, David Sretavan ${ }^{2}$, and Hyuck Choo ${ }^{1}$ \\ ${ }^{1}$ California Institute of Technology, Pasadena, California, USA \\ ${ }^{2}$ University of California, San Francisco, California, USA
}

\begin{abstract}
We demonstrate a compact implantable intraocular pressure sensor with remote optical readout for glaucoma research and patient management. Using broadband white light between 780 $1150 \mathrm{~nm}$, we excite the sensor's pressure-sensitive optomechanical cavity and detect the reflected light, whose optical signature changes as a function of intraocular pressure. When measured inside an artificial pressure chamber that emulates the anterior chamber of the eye, the sensor provided robust measurements of hydrostatic pressures between $10-60 \mathrm{mmHg}$ with an accuracy of $0.15 \mathrm{mmHg}$ and a linearity of 0.97 .
\end{abstract}

\section{INTRODUCTION}

Glaucoma is a leading cause of blindness, affecting an estimated 4 million Americans and 70 million individuals globally [1]. As glaucoma typically affects the elderly, the aging demographic trends indicate that this disease will continue to be an ever-increasing socioeconomic burden to society [1-4]. Elevated intraocular pressure (IOP) is a major risk factor for glaucoma, and IOP monitoring is the single most important clinical management tool [2]. Despite the pervasive use of IOP readings for disease monitoring, and the clinically proven importance of the aggressive lowering of IOP [5-10], current clinical management is primarily based on only periodic snapshots of IOP in the doctor's office obtained every few months based on indirect measurements [11-12]. The inability of patients to easily monitor their own IOPs at different times of the day or during various daily activities, hinder the comprehensive understanding of the IOP profile of individual patients [13] and the possibility of custom-tailored IOP control. In addition to its use as a patient monitoring parameter, IOP is also the standard readout used in glaucoma research. However, achieving an acceptable level of accuracy and repeatability in animal-IOP measurements requires anesthesia and extreme care [14-15]. Conducting such time-consuming measurements in large populations of animals is a major hurdle in glaucoma drug discovery. The need for better IOP monitoring in clinical ophthalmology and in disease research has been widely appreciated, and a number of efforts have been made to develop MEMS-based implantable sensors [16-18]. However, these implants are too large (diameter: 1-3 mm) for use in rodent models (corneal diameter $\sim 3.5$ $\mathrm{mm}$ ), which make up $>90 \%$ of the animal species used in glaucoma research. Sensor implants with such dimensions may also interfere with normal human ocular function and be cosmetically and psychologically unacceptable to patients.

We are developing a highly miniaturized IOP monitoring system using a nanophotonics-based implantable IOP sensor with remote optical readout that can be adapted for both patient and small animal research use. The device's operating principle and configuration are shown in Figures1 and Figure 2, respectively. A remote detector optically excites the pressure-sensitive nanophotonic structure of the IOP-sensing implant placed in the anterior chamber and detects the reflected light, whose optical signature changes as a function of IOP. Optical detection eliminates the need for large, complex inductivecoupling or capacitive-sensing (LC) structures [16-17] and simplifies sensor design. In addition, readout involves 780-1150nm light that is not sensed by the photosensitive neurons in the retina.
The use of precisely engineered nanophotonic components improves the sensor's resolution and sensitivity, increases optical readout distance, and reduces its size by a factor of 10-30 (ultimately down to $50 \mu \mathrm{m}$ in diameter and $25 \mu \mathrm{m}$ in height) over previously reported implants. Its small size and convenient optical readout will allow more frequent and accurate self-tracking of IOP by patients in home settings. In addition, this technology can be adapted for use in automatically monitoring large cohorts of animals to support glaucoma research and drug discovery.
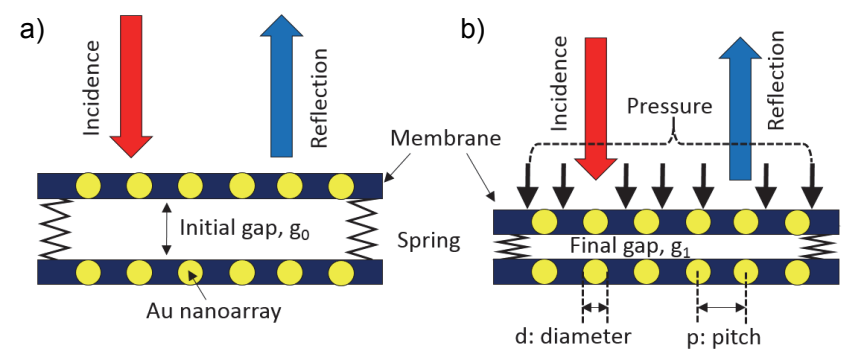

c)

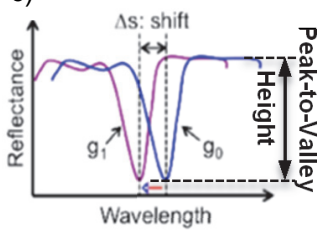

d)

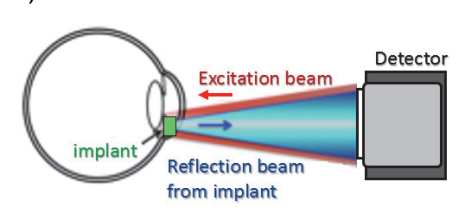

Figure 1: Schematic illustration of the IOP sensor operation. a) Disk-shaped optomechanical cavity whose optical resonance is enhanced by the presence of nanodot arrays. b) Flexible membrane deformation by ambient pressure. c) Resulting shift in resonance due to gap reduction. d) IOP sensor implanted in the anterior chamber and hand-held remote optical reader

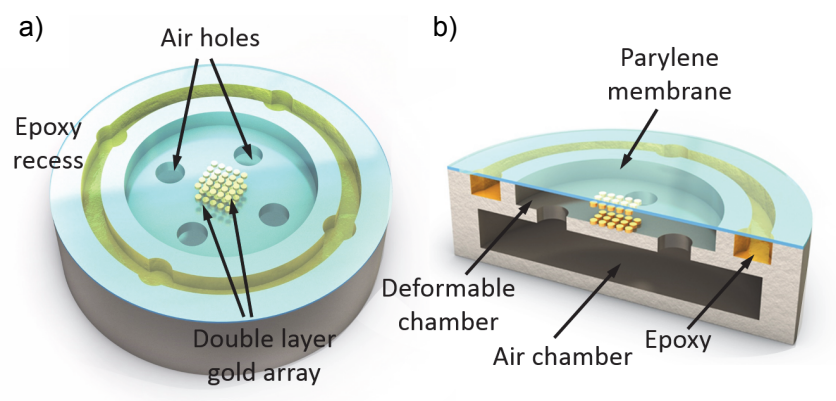

Figure 2: a) 3-D illustration of the sensor. b) Cross-sectional view. The sensor's diameter is $300 \mu \mathrm{m}$, which can be further reduced down to $50 \mu \mathrm{m}$. The size of the nanodot arrays, which determines the active area of the sensor, is $50 \mu \mathrm{m}$ in width and length, and it can also be further reduced down to $25 \mu \mathrm{m}$.

\section{DEVICE DESIGN \\ Operating Principle}

The core of the device, the optomechanical cavity, is formed by a flexible $3-\mu \mathrm{m}$ Parylene membrane with a diameter of $200 \mu \mathrm{m}$ (the deformable part) [16-17] and a rigid silicon substrate on the bottom, Hilton Head Island, South Carolina, June 8-12, 2014 
as shown in Figure 2. Both the Parylene and silicon surfaces are embedded with resonance-enhancing $50 \times 50-\mu \mathrm{m}^{2}$ gold nanodot arrays with the dot-to-dot pitch of $1000 \mathrm{~nm}$ and the nanodot diameter of $800 \mathrm{~nm}$. These values were experimentally determined for the sensor's optimal performance and are discussed in more detail below. As the pressure increases from $1 \mathrm{~atm}$, the initial $2-\mu \mathrm{m}$ gap between the Parylene membrane and the silicon ( $\mathrm{Si}$ ) substrate decreases, and this decrease in the gap shifts the resonant wavelength of the cavity (Fig. 1).

\section{Optomechanical Simulation and Design}

To design a pressure sensor that exhibits highly linear mapping between spectral resonance shifts and pressure changes, we ran a series of optomechanical finite-element-method (FEM) and finitedifference-time-domain (FDTD) simulations using commercial programs (COMSOL and CST), in combination with experimental measurements.

We first determined the spectral range for pressure sensing. The wavelength range of interest is between 770 and $1100 \mathrm{~nm}$ because the light absorption in water and tissues would be minimal in this range, facilitating the penetration through the cornea and the aqueous humor. In addition, this wavelength range is not sensed by photoreceptor cells in the retina. We set the initial cavity gap at 2 $\mu \mathrm{m}$, which is a gap value that can readily be microfabricated. Using FDTD simulation, we first identified a free spectral range of the sensor cavity for clear detection of the resonance shift, as shown in Figure 3.

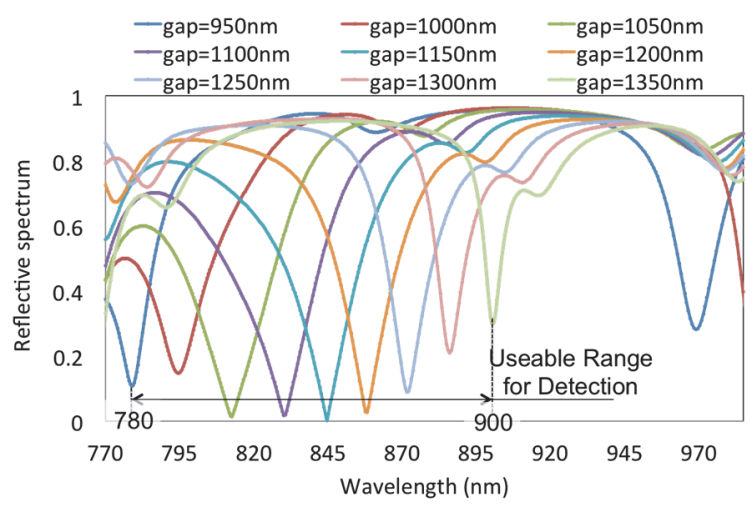

Figure 3: Simulated spectral shift of the cavity resonance as a function of the gap: The usable range is between 780 and 900 or total shift range of $120 \mathrm{~nm}$.

The simulation results have indicated that the free spectral range exists between 780 and $900 \mathrm{~nm}$, which would allow a shift range of $120 \mathrm{~nm}$, when the gap decreases by $400 \mathrm{~nm}$, from $2 \mu \mathrm{m}$ to $1.6 \mu \mathrm{m}$. Next, using FEM simulation, we designed the Parylene membrane - its diameter and thickness - so that it would deflect $400 \mathrm{~nm}$ in response to the change in the ambient pressure (ie. IOP) of $40 \mathrm{mmHg}$ between 10 and $50 \mathrm{mmHg}$. Based on our simulation results, the diameter and the thickness of the Parylene membrane were determined to be $100 \mu \mathrm{m}$ and $3 \mu \mathrm{m}$.

\section{Resonance-Enhancing Nanodot Arrays}

The resonance strength and properties of a Fabry-Perot optical cavity are heavily influenced by the reflectivity at the cavity ends. If the reflectivity is high, the resonance becomes very narrow and sharp, and the quality factor of the resonance increases. However, in this case, the overall amplitude (peak-to-valley height) of the resonance, which directly translates to the signal-to-noise ratio for sensing application, significantly decreases. On the other hand, if the reflectivity is low, the resonance becomes very broad and its amplitude also becomes smaller, making it difficult to detect the resonance. This tradeoff is clearly illustrated by the blue line (from simulation) in Figure 5. Very high or low reflectivity results in low resonance amplitude or peak-to-valley height. An optimal reflectivity exists around $0.6 \pm 0.2$, where the peak-to-valley height is maximized.

a)

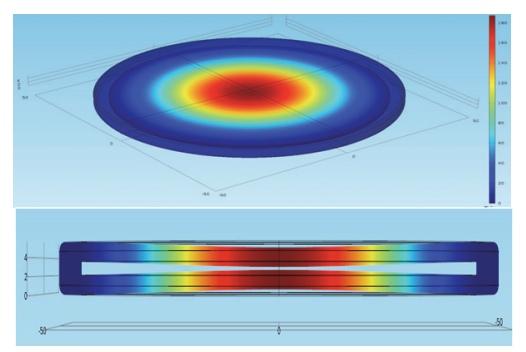

b)
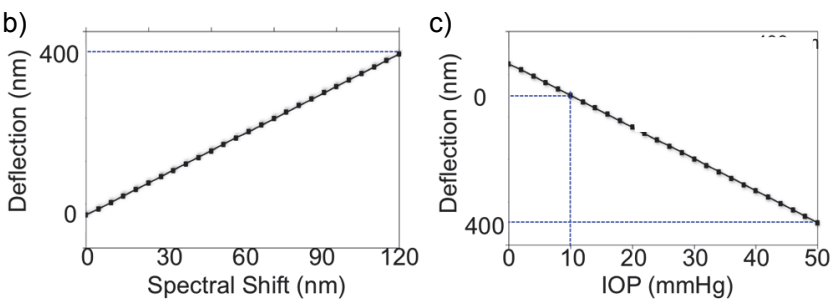

Figure 4: a) FEM simulation of a Parylene membrane deformation as a function of hydrostatic ambient pressure change. b) Linear mapping between "the membrane deflection vs. the spectral shift." c) Linear mapping between "the membrane deflection vs. the intraocular pressure change." For $1 \mathrm{mmHg}$ change, the membrane deforms by $10 \mathrm{~nm}$, and this results in $3 \mathrm{~nm}$ shift in resonance or $3 \mathrm{~nm}$ spectral shift per $1 \mathrm{mmHg}$ pressure change.

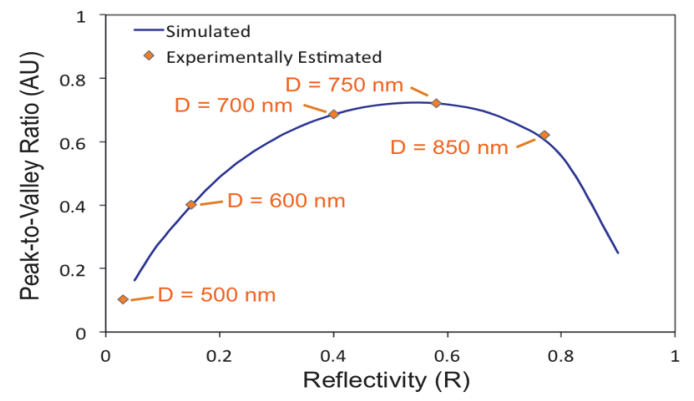

Figure 5: Measured P-V ratios vs. nanodot diameters: The optimal diameter ranges between 700 and $850 \mathrm{~nm}$.

Hence, we use engineered gold nanodot arrays to optimize the reflectivity of the Parylene membrane surface and the silicon surface. In order to adjust reflectivity of a membrane using nanodot arrays, we only need to vary the diameter and pitch of the nanodots, which can be accomplished precisely and repeatedly using an electron-beam lithography system. Consequently, compared to using other coating techniques, the placement of gold nanodots allows us to control Parylene membrane reflectivity in a more reliable and repeatable manner. To find an optimal diameter, we fabricated a series of nanodot arrays with varying diameters from 500 to $850 \mathrm{~nm}$ while keeping the pitch at $1000 \mathrm{~nm}$ and experimentally measured their reflectivities. Fitting the experimentally obtained reflectivities onto the simulation curve as 
shown in Figure 5, we found that the optimal nanodot diameter was $750 \mathrm{~nm}$. To keep our nanofabrication parameters simple, we chose $800 \mathrm{~nm}$ for the nanodot diameter.

\section{DEVICE FABRICATION}

The proposed device is fabricated on a silicon wafer using microscale and nanoscale fabrication techniques. To fabricate the sensor, we first performed photolithography and deep reactive ion etching (DRIE) to create a $2-\mu \mathrm{m}$-deep cavity chamber and a recess (for adhesive) in the Si wafer (Figure 6a). Then, we patterned a 170nm-thick gold nanodot array on the $\mathrm{Si}$ substrate using e-beam lithography, gold evaporation, and lift-off (Figure 6b). Next, using DRIE, we created four air holes on the front side of the wafer and on the air reserve chamber on the back of the wafer (Figure 6c). During the etching process, the air reserve chamber eventually reached the air holes, and they all become connected. Then we patterned another gold nanodot array on the Parylene membrane (Figure 6d) using e-beam lithography, gold evaporation, and lift-off. Next, using epoxy, we assembled the Parylene membrane and the $\mathrm{Si}$ substrate while keeping their nanodot arrays aligned to each other under the optical microscope (Figure 6e). Finally we completely sealed the air reserve chamber using epoxy and a Si chip (Figure 6f).

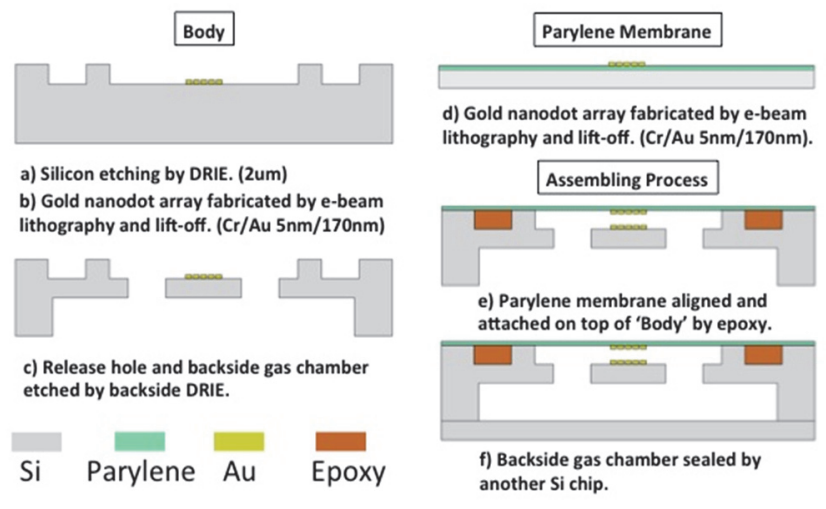

Figure 6: Fabrication process.

The photos of the completed device, including the SEM image of the gold nanodot, are shown in Figure 7. The requirement on alignment accuracy between the two gold nanodot arrays, one on the Parylene membrane and the other on the silicon substrate, is lenient. The maximum misalignment shown in Figure $7 \mathrm{~b}$ is about $4 \mu \mathrm{m}$, and the size of the resulting active sensing area is $85 \%$ of the designed value, which can easily accommodate the probing beam and would not degrade the performance of the sensor.
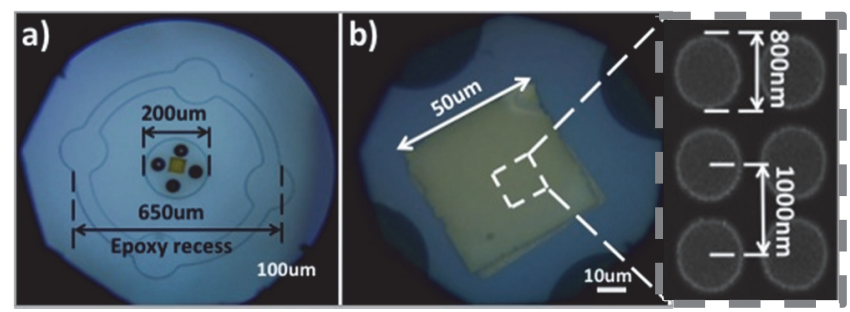

Figure 7: Images of the fabricated device: a) A photo of the entire device (top view). b) Two superimposed gold nanodot arrays. The SEM image shows the gold nanodot array on the Parylene membrane.

\section{DEVICE MEASUREMENTS}

We tested the completed devices inside a water-filled pressure chamber that emulated the anterior chamber of the human eyes (Figure 8). The artificial chamber has a transparent window on the top for optical measurements, and its internal pressure can be precisely adjusted using an external regulator and simultaneously monitored using a digital pressure gauge (model name: DPGWB04; manufacturer: Dwyer Instruments, INC.; accuracy: $\pm 0.5 \%$; thermal effect: $0.05 \%$ ).

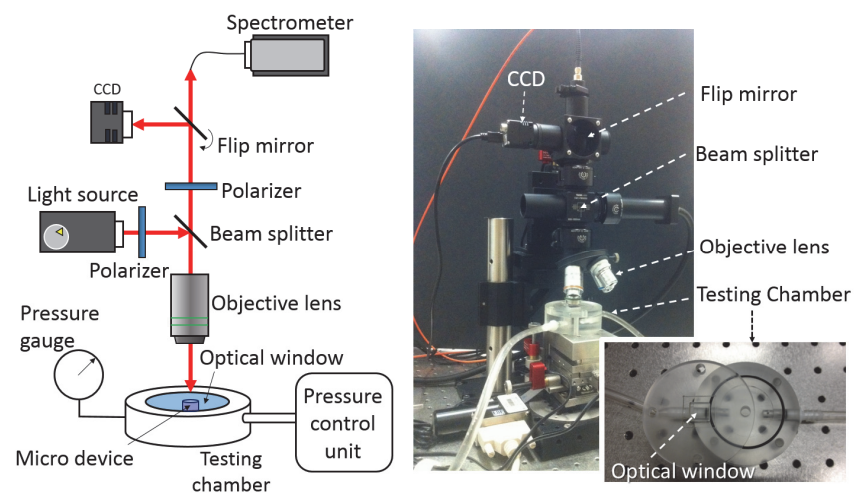

Figure 8: A schematic diagram and a photo of the measurement setup. The inset in the bottom-right corner shows the in-house built pressure chamber that emulates the environment of the anterior chamber.

Using a broadband source (model name: OSL1 High-Intensity Fiber Light Source; manufacturer: Thorlabs) and a commercially available mini-spectrometer (Maya 2000Pro by Ocean Optics; $0.22-$ $\mathrm{nm}$ spectral resolution), we remotely acquired the reflection spectra from the sensor in the chamber at a distance of $7 \mathrm{~mm}$ (Figure 8). As we linearly increased the chamber pressure from 10 to $60 \mathrm{mmHg}$ and tracked the locations of the major peaks in the spectra, we observed that the spectra shifted to the shorter wavelengths, as shown in Figure 9. The average values and standard deviation of the peak locations were determined from over 300 measurements.

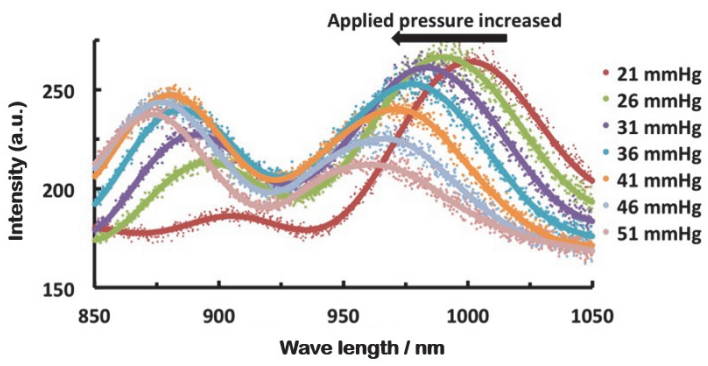

Figure 9: Reflective spectra showing resonance shift as a function of hydrostatic pressure between 21 and $51 \mathrm{mmHg}$.

The mapping between the pressure changes and the resonance shifts was highly linear as shown in Figure 10, with a linear fit of 0.966. The sensitivity and the accuracy of the pressure sensor were 1.53 $\mathrm{nm} / \mathrm{mmHg}$ and $0.15 \mathrm{mmHg}$ over the range of $10-60 \mathrm{mmHg}$. The mini-spectrophotometer with $0.22 \mathrm{~nm}$ spectral resolution was used reliably to resolve $1.53 \mathrm{~nm}$ change in resonance shift per $1 \mathrm{mmHg}$ change in pressure. When compared to the sensor without gold nanodot arrays, the presence of the nanodot array significantly enhanced the signal-to-noise ratio of the sensor by a factor of seven and enabled more robust remote detection. 


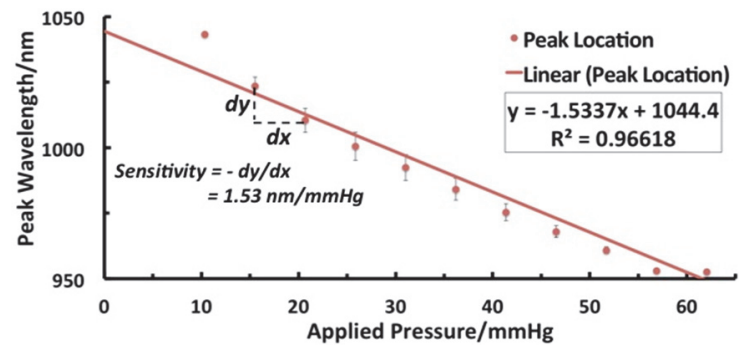

Figure 10: Linear mapping between the shift in peak locations and the ambient hydrostatic pressure: the linearity is 0.96618 .

Following the successful measurements in the artificial pressure chamber, we pursued measurements in ex-vivo rabbit eyes. Figure 11 shows robust optical resonance captured remotely over a 7-mm distance from an earlier prototype device (consisting of two rigid membranes that do not flex) implanted in the anterior chamber of an ex-vivo rabbit eye and also in saline as a reference measurement. In both cases, we obtained consistent resonance peaks at identical locations.

a)

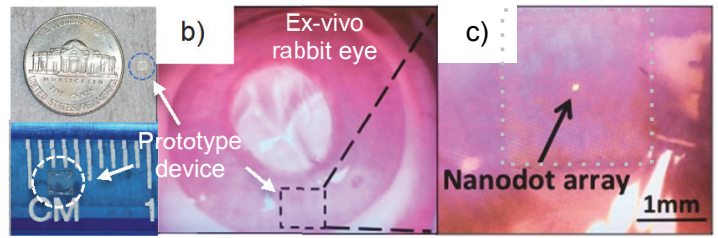

d)

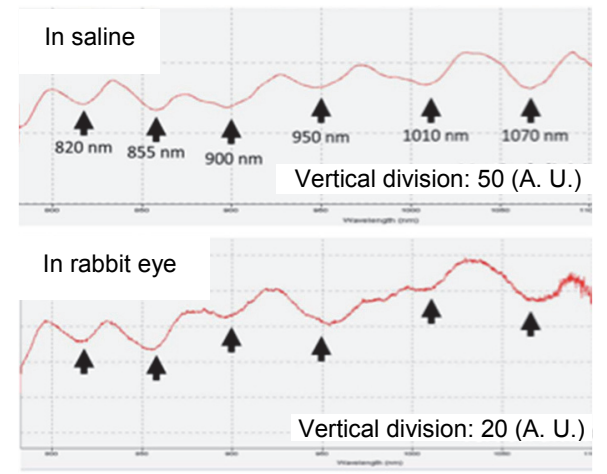

Figure 11: a) Earlier prototype device, $\sim 2 \mathrm{~mm}$ by $2 \mathrm{~mm}$. b) Prototype implanted in the anterior chamber of an ex-vivo rabbit eye. c) A zoom-in image of the implant in the ex-vivo rabbit eyethe gold nanodot array appears as a bright spot in the image, indicated by the arrow. d) A resonance spectrum captured from the prototype device implanted in ex-vivo rabbit eye and also in saline. The resonance dips appear exactly at the identical locations.

\section{CONCLUSIONS}

We have designed and fabricated a compact implantable IOP sensor with remote optical readout for glaucoma research and patient management. We have demonstrated its IOP-sensing capability between 10-60 $\mathrm{mmHg}$ in an artificial pressure chamber and captured the resonance spectrum from a prototype sensor implanted in the anterior chamber of an ex-vivo rabbit eye. We are presently working on (1) measurements in ex-vivo and in-vivo rabbit eyes; (2) further miniaturization of the implantable IOP sensors below $100 \mu \mathrm{m}$ for use in rodent eyes; (3) a longer optical readout distance (theoretical limit $\sim 20 \mathrm{~cm}$ ); and (4) algorithms for automatic assignments of spectral shifts to pressure values.

\section{REFERENCES}

[1] www.glaucoma.org (Glaucoma Research Foundation)

[2] J. C. Morrison and I. P. Pollock, Glaucoma: Science and Practice, New York, New York: Thieme, 2002, Ch.1 \& Ch.5.

[3] www.aoa.gov (Administration on Aging, Department of Health \& Human Services)

[4] www.nei.nih.gov (National Eye Institute, NIH).

[5] B. L. Lee and M. R. Wilson, "Ocular Hypertension Treatment Study (OHTA) commentary," Current Opinion in Ophthalmology, vol. 14, no. 2, pp. 74-77, 2003.

[6] M. C. Leskea, A. Heijl, L. Hyman, B. Bengtsson, and E. Komaroff, "Factors for progression and glaucoma treatment: the Early Manifest Glaucoma Trial," Current Opinion in Ophthalmology, vol. 15, no. 2, pp. 102-106, Apr. 2004.

[7] J. T. Wilensky, "The role of diurnal pressure measurements in the management of open angle glaucoma," Current Opinion in Ophthalmology, vol. 15, no. 2, pp. 90-92, Apr. 2004.

[8] H. Bagga, J. H. Liu, and R. N. Weinreb, "Intraocular pressure measurements throughout the 24h," Current Opinion in Ophthalmology, vol. 20, no. 2, pp. 79-83, Mar. 2009.

[9] S. Asrani, R. Zeimer, J. Wilensky, D. Gieser, S. Vitale, and K. Lindenmuth, "Large diurnal fluctuations in intraocular pressure are an independent risk factor in patients with glaucoma,” J. Glaucoma, vol. 9, no. 2, pp. 134-142, Apr. 2000.

[10] E. Hughes, P. Spry, and J. Diamond, "24-hour monitoring of intraocular pressure in glaucoma management: A retrospective review," J. Glaucoma, vol. 9, no. 2, pp. 232-236, Jun. 2003.

[11] C. R. Ethier, M. Johnson, and J. Ruberti, "Ocular biomechanics and biotransport," Annual Review of Biomedical Engineering, vol. 6, pp. 249-273, 2004.

[12] I. G. Pallikaris, G. D. Kyomionis, H. S. Ginis, G. A. Kounis, and M. K. Tsilimbaris, "Ocular rigidity in living human eyes," IOVS, vol. 46, no. 2, pp. 409-414, Feb. 2005.

[13]K. Singh and A. Shrivastava, "Intraocular pressure fluctuations: how much do they matter?" Current Opinion in Ophthalmology, vol. 20, no. 2, pp. 84-7, Mar. 2009.

[14] L. Jia, W. O. Cepurna, E. C. Johnson, and J. C. Morrison, "Effect of General Anesthetics on IOP in Rats with Experimental Aqueous Outflow Obstruction," IOVS, vol. 41, no. 11 , pp. 3415-3419, Oct. 2000.

[15] J. Danias, A. I. Kontiola, T. Filippopoulos, and T. Mittag, "Method for the Noninvasive Measurement of Intraocular Pressure in Mice," IOVS, vol. 44, no. 3, pp. 1138-1141, Mar. 2003.

[16] P. J. Chen, D. C. Rodger, R. Agrawal, S. Saati, E. Meng, R. Varma, M. S. Humayun, and Y. C. Tai, "Implantable micromechanical parylene-based pressure sensors for unpowered intraocular pressure sensing," J. Micromechanics and Microengineering, vol. 17, pp.1931-1938, Oct. 2007.

[17] P. J. Chen, S. Saati, R. Varma, M. S. Humayun, and Y. C. Tai, "Wireless Intraocular Pressure Sensing Using Microfabricated Minimally Invasive Flexible-Coiled LC Sensor Implant," J. Microelectromechanical Systems, vol. 19, no. 4, pp. 721-734, Aug. 2010.

[18] D. Ha, W. N. de Vries, S. W. M John, P. P. Irazoqui, W. J. Chappell, "Polymer-based miniature flexible capacitive pressure sensor for intraocular pressure (IOP) monitoring inside a mouse eye," Bioemdical Microdevices, DOI 10.1007/s10544-011-9598-3, published online, Oct. 2011.

\section{CONTACT}

*J. O. Lee, tel: +1-626-395-8870; jolee@caltech.edu 